\title{
Implant failure following pedicle based dynamic stabilization of the lumbar spine
}

\author{
Hans Schauvliege, Marc Du Bois, Jan Verlooy
}

From the department of orthopedic surgery of the Heilig Hartziekenhuis Mol, Mol, Belgium

Pedicle-based dynamic stabilization (PBDS) devices such as Dynesys are promoted as an alternative and less invasive option for rigid stabilization of one and even more levels of the lumbar spine. Promising features of the Dynesys system, as well as shortcomings, became obvious in several clinical studies. Since 2012, we started using a new PBDS device as an alternative for the Dynesys, to avoid the screw loosening and the kyphosing effect.

The objective is to compare failure rates between the Dynesys and Balan-C type PBDS implant and factors affecting outcome.

In a retrospective study we investigated a total of 90 patients with lumbar pedicle screw dynamic stabilization (a group of 64 patiënts with Dynesys stabilization is compared to a group of 26 patients with Balan-C stabilization). Mean follow-up was 48 and 38 months, respectively. Using logistic regression analysis the impact of baseline characteristics such as gender, age, body mass index (BMI), indication for surgery, primary or revision surgery, single versus more level surgery, surgeon's experience and type of the implant on implant failure was analyzed.

We found a statistically significant difference in failure rates between the two systems $(13 \%$ in the Dynesys group versus $62 \%$ in the Balan-C group). In multivariate analysis, type of implant was associated with implant failure (odds ratio : 13).

Our current results call for an optimization of the pre-and post-marketing surveillance of pedicle-based dynamic stabilization.

Keywords : medical device; pedicle based dynamic stabilization; market authorization; failure ; product liability.

\section{INTRODUCTION}

The last two decades, the range of lumbar spine pedicle screw stabilization techniques was expanded with more dynamic and hybrid techniques. The Dynesys system is promoted as a less invasive technique for stabilization of one and even more levels $(1,2,3,4,5)$. This system offers the possibility to restore disc height and to preserve some mobility. The Dynesys system consists of polycarbonate urethane spacers to limit spinal extension and a polymer cord acting as a tension band to limit spinal flexion combined with a pedicle screw system. It also exists in a dynamic transition option (DTO) variant allowing a traditional rigid fixation at the lower level combined with a dynamic stabilization at the upper level. The Dynesys system was introduced in spine surgery by Dr. Gilles Dubois

Hans Schauvliege ${ }^{1}, \mathrm{MD}$,

- Jan Verlooy², MD., PhD,

- Marc Du Bois 3 , MD., PhD

${ }^{1}$ Department of Orthopedic Surgery, Heilig Hartziekenhuis

Mol, Gasthuisstraat 1, $2400 \mathrm{Mol}$, Belgium.

${ }^{2}$ Departement of Social Medecine, Antwerp University Faculty of Medecine, Campus Drie Eiken, Universiteitsplein 1, D.R.228, 2610 Wilrijk, Belgium.

${ }^{3}$ Departement of Environmental Health, Faculty of Medecine, Catholic University of Leuven, Kapucijnenvoer 35 blok d-bus 7001, 3000 Leuven, Belgium

Correspondence : Schauvliege Hans, Phone : +3214712055.

Email : hans.schauvliege@telenet.be

- 2021, Acta Orthopædica Belgica. 
from Toulouse. This system can rely on a follow-up of 22 years of clinical experience $(1,2,3,4,5)$. In 2012 we launched the Balan-C system in our department as a promising alternative for the Dynesys system to avoid the problem of screw loosening and the kyphosing effect $(1,2,4)$. The Balan-C system consists of Polyether ether ketone (PEEK) rods with silicone bumpers, combined with a pedicle screw system. The PEEK-rod-silicone construct is acting as a hinge, allowing a limited motion of the functional spinal unit. This system also exists in different types of lordotic curves and in a DTO variant. To our knowledge, no long-term results are available about the Balan-C system (7). This study will focus on the outcome of the Balan-C system compared to the Dynesys implant.

\section{MATERIALS AND METHODS}

A total of 90 patients with lumbar pedicle screw dynamic stabilization surgery between January 1, 2006, and December 31, 2015, was investigated in our department of orthopedic surgery (a group of 64 patients with a stabilization of the Dynesys type compared to a group of 26 patients with a stabilization of the Balan-C type). All patiënts were operated by the same surgeon. We investigated the impact of baseline characteristics such as gender, patiënt age at the time of the intervention, BMI, indication for stabilization, primary or revision surgery, single versus more level construct and years of experience with stabilizing in case of failure. The aim of the present study was to compare the failure rate between the Dynesys group and the Balan-C group. Characteristics of patiënts in the Dynesys and Balan-C group were compared using the KruskalWallis test for continuous and the Chi-Square test for categorical variables. Association of gender, age, BMI, indication for surgery, revision surgery, more level surgery, surgeon's experience and type of implant with implant failure was analyzed through logistic regression analysis. All statistical analyses were performed using SPSS version 24 .

\section{RESULTS}

The failure rate in the Dynesys group was $13 \%$, in accordance with previous research $(1,2,5)$.
In the Dynesys group, 8 revisions (13\%) were performed, including 7 for screw loosening and 1 for screw breakage following trauma, several years after surgery in a patiënt with a previously favorable result. The failures occurred all in more level constructs. In the Balan-C group, we found 16 revisions (62\%). One revision involved the symptomatic aseptic inflammation around the implant in a hybrid construct. Laboratory research and PET-scan showed signs of infectious process on the implant. Intraoperative culture did not reveal any pathogens. Favorable MRI findings were found both at the fusion and transition level. Another revision related to screw loosening was done in a hybrid construct with satisfactory NMR findings at the fusion-level as well as on the transition level. In either case, the implant could be removed safely.

Revision surgery was needed for rod breakage in 14 cases. In all cases, dynamic stabilization was converted to conventional fusion. Average hospital stay was 2 days, considering it concerned only a partial replacement of the dynamic implant by a rigid rod. Two revisions for breakage were performed for acute back pain after a fall and following a car accident. In both cases, breakage of the connector was diagnosed on $\mathrm{CT}$ imaging with reconstruction in 3 planes (3D CT-scan). Before the causative trauma, there was no clinical or radiological evidence of implant failure.

The implant breakage occurred always in the connecting rod section of the Balan-C implant. Breakage was bilateral in 13 of the $14(93 \%)$ cases and 8 times (30\%) at the rod portion, and in 19 times $(70 \%)$ at the dynamic bumper portion. Breakage was found in $3(21 \%)$ single and $11(79 \%)$ double level constructs. The Balan-C system led to a failure rate of $62 \%$ (Fig. 1 : breakage of a Balan-C implant through the bumper portion; Fig. 2: breakage through the rod portion).

Statistical analysis of baseline characteristics could not reveal a significant difference between the two groups except for surgical experience. This finding can be explained through the history of both series : the Balan-C started since 2012 as an alternative to the Dynesys technique (table 1).

Using logistic regression analysis, the type of the implant was the only parameter that showed 


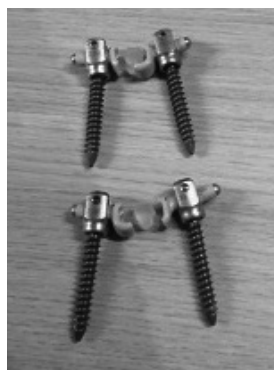

Fig. 1. - Breakage of a Balan-C implant through the bumper portion.

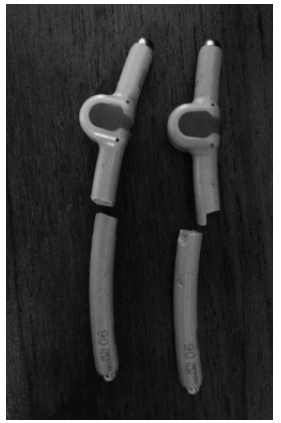

Fig. 2. - Breakage through the rod portion.

significant impact on the outcome. Hence, we concluded that the failure rate is purely implantrelated (table 2).

We had to rely on more targeted medical imaging techniques to demonstrate implant breakage.

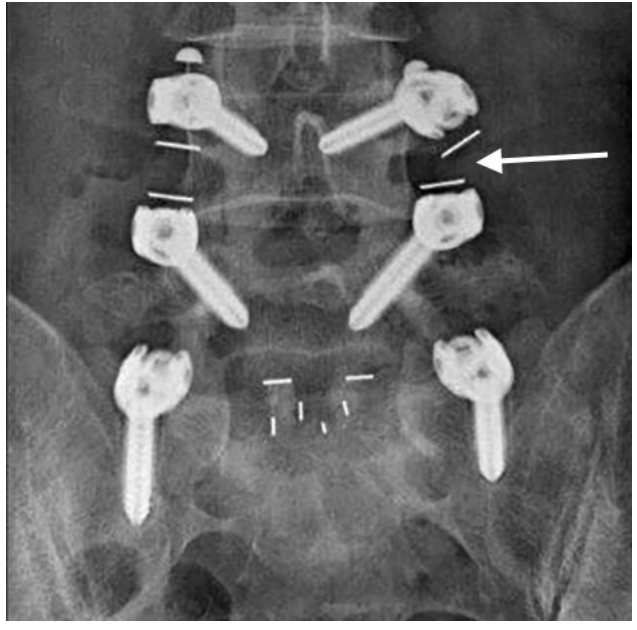

Fig. 3. - Implant failure documented on conventional X-ray image by the asymmetric aspect of the left-sided marker in the bumper portion

Of the 8 failures in the Dynesys group, $3(38 \%)$ failures were confirmed by X-ray and $4(50 \%)$ by CTscan. Implant failure was not visible on conventional imaging in only 1 patiënt (12\%) though failure was suspected due a huge recurring herniated disc on the stabilized segment. The failure was per-operatively discovered as being caused by loosening of the

Table I. - Baseline Characteristics

\begin{tabular}{|l|c|c|c|}
\hline Characteristics : & Dynesys & Balan-c & P \\
\hline Age (mean,yr) & 48 & 39 & 0.105 \\
\hline Gender male & $35(55 \%)$ & $13(50 \%)$ & 0.686 \\
\hline BMI mean & 44 & 48 & 0.553 \\
\hline Indication : disc hernia/disc degeneration : dh & $34(53 \%)$ & $9(35 \%)$ & 0.111 \\
\hline Primary/revision : primary & $50(78 \%)$ & $22(85 \%)$ & 0.485 \\
\hline Single or more level surg. : more level & $50(78 \%)$ & $21(81 \%)$ & 0.781 \\
\hline Experience : (mths) (mean) & 35 & 71 & 0,000 \\
\hline Follow-up : (mths) (mean) & 48 & 38 & 0,097 \\
\hline Failed or not failed : failed & $\mathbf{8 ( 1 3 \% )}$ & $\mathbf{1 6}(\mathbf{6 2} \%)$ & $\mathbf{0 . 0 0 0}$ \\
\hline
\end{tabular}

Table II. — Logistic regression model with 8 variables correlated to failure

\begin{tabular}{|l|c|c|c|}
\hline Variables : & Odds ratio & 95\% C.I. lower & 95\% C.I. higher \\
\hline Gender & 0.679 & 0.190 & 2,433 \\
\hline Age & 1,000 & 0.948 & 1,055 \\
\hline BMI & 1,030 & 0.900 & 1,178 \\
\hline Indication: DH/DD & 3,208 & 0.843 & 12,206 \\
\hline Primary/revision & 0.684 & 0.175 & 2,990 \\
\hline One or more level surg. & 1,546 & 0.259 & 9,238 \\
\hline Experience (Y) & 0.966 & 0.662 & 1,409 \\
\hline Type of surgery & 12,825 & 2.310 & 71,212 \\
\hline
\end{tabular}




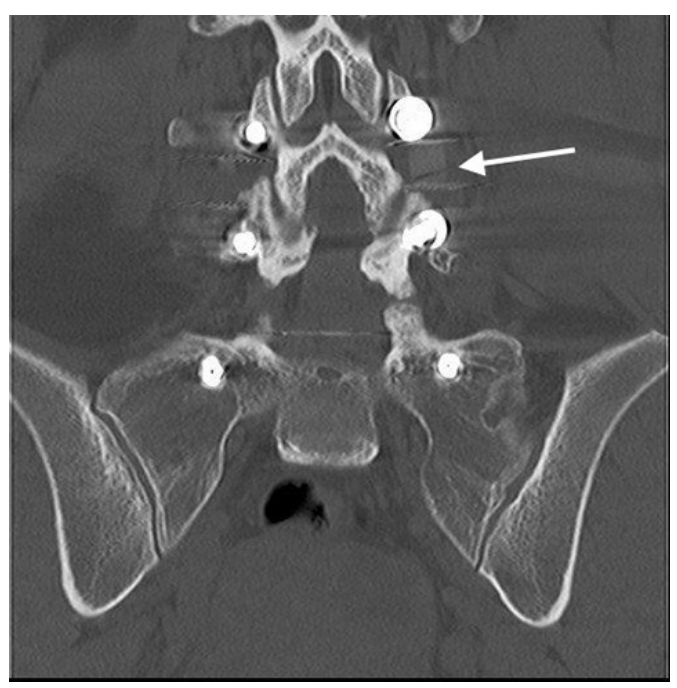

Fig. 4. - Implant failure (see arrow) documented on 3D CT-scan

Dynesys screws. In the Balan-C group failure arising from rod breakage was confirmed by X-ray in 4 (29\%) patiënts and by CT-scan in another 4 (29\%) cases. All cases needed a 3D CT-scan to confirm the broken Balan-C rod component. Breakage was not visible on X-ray or 3D CT-scan in 6 patiënts (42\%) where it was clinically suspected and confirmed peroperatively. (Fig. 3 : implant failure documented on conventional X-ray image by the asymmetric aspect of the left-sided marker in the bumper portion; Fig. 4 : implant failure (see arrow) documented on 3D CT-scan).

\section{DISCUSSION}

Our results showed a worse result for the Balan-C system. To our knowledge, a failure rate of that size has never been reported before in literature. Only one recent study described the radiological features of a new pedicle based dynamic stabilization (PBDS) system and reports implant breakage in $27 \%$ of the cases which is considered as striking failure (10 out of 37 interventions) and lead to retrieval of the implant from the market. The PBDS technique concerned the CD-Horizon Agile system, which has some similar features with the Balan-C system. Consistent with our findings, the authors reported a breakage in the dynamic spacer section of the implant (6). In a recent 2-year follow-up study with the Balan-C system, no screw loosening or breakage was reported (7). As opposed to our current practice, postoperative imaging was limited to conventional. In our series, breakage was not visible on conventional X-ray in 10 of the $14(71 \%)$ cases and the mean time to breakage was 30 months, which may explain the difference in outcome. This finding underscores the importance of a regular follow-up until one year postoperatively followed by a yearly follow-up. In cases with an innovative implant we propose a 5-year follow-up period. Our results are in disagreement with present scientific evidence about PBDS-systems (1,2,3,5).

We scrutinized in a report of the Belgian Health Care Knowledge Centre on the Dynesys system. Promising features of the Dynesys system as prevention of adjacent-level-degeneration have been critically considered. One 4-year followup study failed to demonstrate the property of the Dynesys system to prevent adjacent level degeneration. Complications included a revision rate of $21 \%$ and a screw loosening rate of $11 \%$ (5). These findings are in accordance with Schroeder et al (3). In a recent review failure and iterative surgeries rates following a Dynesys implant were similar to conventional rigid pedicle-screw systems. Apparently, the rate of adjacent segment degeneration (ASD) appeared to be lower supporting the theory of reduced biomechanical stress. The screw loosening rate was $12 \%$. Implant failure, such as spacer and screw breakage was rare (1).

A recent systematic literature review about PBDS-systems, in general, found adjacent segment degeneration in 0 to $30 \%$. Revision surgery for breakage was performed in $9 \%$. Technical failures were design related but also linked with patientrelated properties such as poor bone quality. Surgeon's adherence to strict indications as moderate degeneration, mild degenerative facet arthritis, low-grade spondylolisthesis, segmental instability and dynamic stenosis is of paramount importance. The sagittal balance of the vertebral column and the degree of instability deserve special emphasis $(2,4,8)$. The posterior shift of the center of rotation is an important feature of dynamic stabilization. PBDS- systems must be able to withstand shear forces to prevent failure $(2,9,10,11)$. 
The literature about PEEK-rods is extremely scarce. PEEK-rods proved both an advantage and a drawback to spinal stabilizing. The advantages include better load-sharing with the lateral column, the slightly greater flexibility of the PEEK-rod biomechanically beneficial in terms of fusion and lower risk of screw breakage at the expense of an increased risk of PEEK-rod breakage $(2,7,12,13)$. In the Balan- $\mathrm{C}$, there is a combination of a PEEK-rod with a PEEK-silicone bumper. In our series, we noted $8(30 \%)$ breakages of the rod and $19(70 \%)$ of the bumper. There is nothing in the literature up-todate about this. The composite nature of the Balan-C implant does not allow to draw any conclusion on PEEK rods in general.

Though our study was retrospective, the strength of our study includes the large sample size and that the intervention was performed by the same surgeon. Given the young average age of the Balan-C group (39 years), we did not investigate bone quality.

Our study has some policy implications :

The regulatory framework for obtaining market access for high-risk medical implants in Europe differs strongly to the United States where this Balan-C system was never allowed. In the United States, RCT's are a necessary tool in obtaining market access. To gain market access in Europe, studies that prove safety and performance are mandatory and RCT's are not necessary $(14,15,16)$. Safety studies are carried out by notified bodies selected by the manufacturer which establishes a relationship of dependency. European healthcare providers are not always aware of the weakness in the pre-clinical evaluation of these implantable medical devices not providing the same level of safety as drugs (16). Testing of new innovative devices should be entrusted to high volume centers with sufficiënt expertise. Reinforcing post-market surveillance is equally necessary. Council Directive 93/42/EEC of 14 June 1993 on medical devices is a European harmonization law that is transposed into Belgium legislation. Pursuant to article 11 of The Royal Decree of March 18, 1999, the manufacturer, the distributor, the notified body and (para)-medical practitioners have the duty to inform the Federal Agency for Medicines and Health Products (FAMHP) in case of incidents $(7,16)$.
Practice has shown that the Balan-C system was withdrawn from the market after numerous revision surgeries for implant failure. Literature is skeptical on spontaneous reporting of adverse events by the manufacturer, the individual spine surgeon or the clinical centre (in less than $0.5 \%$ of the adverse events) (16).

Legislation on medical implants has been evolving since April the 5th of 2017 when the European Commission adopted the proposal for two regulations on medical devices (MD), which "establish a modernized and more robust EU legislative framework to ensure better protection of public health and patient safety" (17). Key points of this regulation are the strengthening of the control over the notified bodies, who are allocated with the obligatory task to participate in the annual audits, and the unannounced postmarket surveillance audits. A European electronic MD database and a unique device identification system will be accessible to healthcare providers and in a simplified version to the public. This new European regulation on MD's is a positive step in preventing striking failure of medical devices and provides for both surgeon and patiënt high-quality care and safety. The publicly accessible database facilitates informed consent $(17,18,19,20)$. Pending its entry into force, the systematic recording in a spine registry such as 'Spine Tango', is encouraged (21,22). Furthermore, the Belgian law on product liability provides for the manufacturer's liability in case of medical device failure. The producer's liability ends 10 years following product marketing. A customer suffering injury may submit a complaint within 3 years from the day when the damages were incurred. The producer can invoke the development risk as a defense $(23,24)$.

A unique finding in the Balan- $\mathrm{C}$ cases is diagnose of product failure is often belated for lack of clear evidence on imaging. In some cases following per operative confirmation, implant failure became apparent. We recommend 3D CT-scan to investigate unexplained pain in patiënts with PBDS implants, especially those with PEEK-silicone composition.

Socioeconomic aspects of implant failure were not included in our investigation. However, it is obvious that the impact of revision surgery has an 
important burden on healthcare in terms of hospital stay and absenteeism. In addition, implant breakage in road accident victims is likely to prove contentious in view of causation analysis. We conclude with following concerns : Breakage of PBDS implants is a main issue for spine surgeons. A regular followup during the first postoperative year, followed by a yearly checkup in the next 5 years is recommended. Unexplained pain following PBDS-stabilization requires $3 \mathrm{D}$ CT scan for further exploration.

\section{REFERENCES}

1. Pham MH, Mehta VA, Patel NN. et al. Complications associated with the Dynesys dynamic stabilization system : a comprehensive review of the literature. Neurosurg. Focus 2016 ; 40(1) : E2.

2. Prud'homme M, Barrios C, Rouch P, Charles YP, Steib JP, Skalli W. Clinical Outcomes and Complications After Pedicle-anchored Dynamic or Hybrid Lumbar Spine Stabilization. A Systematic Literature Review. J. Spinal Disord. Tech. 2015 ; 28(8) : E439-E448.

3. Schroeder GD, Murray MR., Hsu WK. A Review of Dynamic Stabilization in the Lumbar Spine. Oper. Techn. Orthop. $2011 ; 21(3): 235-239$

4. Legaye J. Unfavorable influence of the dynamic neutralization system on sagittal balance of the spine. Rev. Chir. Orthop. Repar. Appar. Mot. 2005 ; 91(6) : 542-550.

5. Stordeur S, Gerkens S, Roberfroid D. Interspinous implants and pedicle screws for dynamic stabilization of lumbar spine : Rapid assessment. KCE reports 2009 ; 116C https://kce.fgov.be/sites/default/files/page_documents/ d20091027346.pdf (100517).

6. Hoff E, Strube P, Rohlmann A, Gross C, Putzier M. Which Radiographic Parameters Are Linked to Failure of a Dynamic Spinal Implant? Clin. Orthop. and Relat. R. 2012 ; 470(7) : 1834-1846.

7. Formica M, Cavagnaro L, Basso M, Zanirato A, Felli L, Formica C. (2015) Is it possible to preserve lumbar lordosis after hybrid stabilisation? Preliminary results of a novel rigid-dynamic stabilisation system in degenerative lumbar pathologies. Eur. Spine J. 2015 ; 24 (7Suppl) S849-S854.

8. Kumar M, Baklanov A, Chopin D. Correlation between sagittal plane changes and adjacent segment degeneration following lumbar spine fusion. Eur. Spine J. $2001 ; 10$ : 314-319.
9. Niosi CA, Zhu QA, Wilson DC, Keynan O, Wilson DR, Oxland TR. Biomechanical characterization of the threedimensional kinematic behaviour of the Dynesys dynamic stabilization system : an in vitro study. Eur. Spine J. 2001 ; 10(4) : 314-319.

10. Galbusera F, Bellini CM, Anasetti F, Ciavarro C, Lovi A, Brayda-Bruno M. Rigid and flexible spinal stabilization devices: A biomechanical comparison. Med. Eng. Phys. $2011 ; 33(4)$ : 490-496.

11. Xu HZ, Wang XY, Chi, YL. et al. Biomechanical evaluation of a dynamic pedicle screw fixation device. Clin. Biomech. 2006 ; 21(4) : 330-336

12. Ponnappan RK, Serhan H, Zarda B, Patel R, Albert T, Vaccaro AR. Biomechanical evaluation and comparison of polyetheretherketone rod system to traditional titanium rod fixation. The Spine Journal 2009 ; 9(3) : 263-267.

13. Turner JL, Paller DJ, Murrell CB. (2010) The mechanical effect of commercially pure titanium and polyetheretherketone rods on spinal implants at the operative and adjacent levels. Spine 2010 ; 35(21) : E1076-82.

14. Moojen WA, Bredenoord AL, Viergever RF, Peul WC. (2014) Scientific Evaluation of Spinal Implants : An Ethical Necessity. Spine 2014 ; 39(26) : 2115-2118.

15. Hulstaert F, Neyt M, Vinck I. et al. Pre-market clinical evaluations of innovative high-risk medical devices in Europe. International J. Technol. Assess. Health Care 2012 ; 28(3) : 278-284.

16. Resnic FS, Normand SL. Postmarketing Surveillance of Medical Devices - Filling in the Gaps. New Engl. Med. 2012 ; 366(10) : 875-877 http://europa.eu/rapid/pressrelease_IP-17-847_en.htm (220417)

17. http://europa.eu/rapid/press-release_IP-17-847_en.htm

18. Fouretier A, Bertram D. New regulations on medical devices in Europe: what to expect? Expert Rev. Med. Devic. $2014 ; 11(4)$ : 351-359.

19. https : //osha.europa.eu/nl/legislation/directives/councildirective-85-374-eec (150417)

20. http : //ec.europa.eu/growth/sectors/medical-devices/ regulatory-framework/revision_en (050417)

21. Melloh M, Staub L, Aghayev E. et al. The international spine registry SPINE TANGO : status quo and first results. Eur. Spine J. 2008 ; 17 : 1201-1209 http : //www.eurospine. org/spine-tango.htm (210817).

23. Wuyts D. The Product Liability Directive : More Than Two Decades of Defective Products in Europe. J. Eur. Tort. Law $2014 ; 5: 1-1$ at 9.

24. Arbour ME. Portrait of development risk as a young defence.(product liability) (Technological Innovation and Civil Responsibility) McGill Law J. 2014 ; 59(4) : 913(30). 